(c) Auteure. Cette œuvre, disponible à

http://dx.doi.org/10.18162/fp.2020.542, est distribuée sous licence Creative Commons Attribution 4.0 International http://creativecommons.org/licences/by/4.0/deed.fr

Marie-Hélène Forget
Université du Québec à Trois-Rivières (Canada)

\title{
Sur quels savoirs des stagiaires en enseignement du français au secondaire appuient-ils leurs choix didactiques?
}

What knowledge do French education secondary school trainees provide for their didactic choices?

\section{ésumé}

Bien que les savoirs didactiques (savoirs à et pour enseigner) fondent l'acte d'enseigner, peu de recherches s'intéressent à la mobilisation de ces savoirs par des stagiaires en enseignement du français. Cette recherche exploratoire, réalisée au Québec en 2017, fait état du type et de la qualité des savoirs didactiques sur lesquels vingt stagiaires en enseignement du français au secondaire appuient leurs choix didactiques dans leurs préparations

de cours. Les résultats montrent une proportion

intéressante de savoirs didactiques de référence par rapport aux savoirs d'expérience, mais un décalage entre ces savoirs et leur actualisation dans les activités conçues et décrites par ces stagiaires.

\section{Mots-clés}

Savoirs didactiques, stagiaires, préparations de cours, enseignement du français, secondaire.

\section{Abstract}

Although didactic knowledge (curriculum and knowledge for teaching) is the foundation of teaching, there is little research on the didactic knowledge of French language teaching trainees and their mobilization in the context of an internship. This exploratory research, conducted in Quebec in 2017, reports on the type and quality of didactic knowledge on which twenty trainees in French secondary education support their didactic choices in their course preparation. The results show an interesting proportion of didactic knowledge compared to the knowledge of experience, but a gap between these didactic knowledge and how they are represented in the activities.

\section{Keywords}

Didactic knowledge, trainees, course preparation, French education, high school.

\section{Cadrage de la recherche}

Les savoirs professionnels sont fondamentaux dans l'exercice de la profession enseignante et garants de la qualité de l'enseignement offert et des apprentissages réalisés par les élèves (Gauthier, Desbiens, Malo, Martineau et Simard, 1997). Parmi les savoirs à développer dans le cadre de la formation initiale en enseignement, mentionnons les savoirs didactiques. Construits au cours de la formation initiale (et ensuite dans l'exercice de la profession), ces savoirs sont fondateurs de l'acte d'enseigner parce qu'ils assurent, entre autres, la mise en œuvre des compétences de planification, de pilotage des situations d'apprentissage, ainsi que l'institutionnalisation adéquate des objets d'enseignement.

Au Québec, on s'intéresse encore peu aux savoirs didactiques effectivement construits dans le cadre de la formation initiale en enseignement du français au secondaire. On sait par exemple que la maitrise des genres discursifs que les stagiaires auront à enseigner nécessite une formation dédiée et un accompagnement (Gagnon,2014; Lord et Sauvaire, 2014). On sait aussi que les savoirs grammaticaux mobilisés lors du pilotage de situations d'apprentissage en contexte de stage sont fragiles (Gauvin et al.,2017). Par ailleurs, si les stagiaires en enseignement primaire sollicitent assez peu les cadres didactiques au moment de préparer les cours qu'ils piloteront en stage au profit des aspects relatifs au climat de la classe (Araujo-Oliveira, Lisée, Lenoir et Maubant, 2011), il en va de même en Europe, où Scheepers (2014) a mis en lumière le caractère occulté des savoirs spécifiquement didactiques dans des préparations de cours de centaines de stagiaires en enseignement du français au secondaire. 
Pourtant, au moment de « concevoir des situations d'enseignement-apprentissage pour les contenus à faire apprendre »(Gouvernement du Québec, 2001, p. 75), l'enseignant (ou le stagiaire) doit :

- sélectionner et interpréter les savoirs disciplinaires [à enseigner] (Gouvernement du Québec, 2001, p. 79);

- choisir des approches didactiques variées et appropriées au développement des compétences visées dans le programme de formation (Gouvernement du Québec, 2001, p. 82);

- appuyer le choix de ses interventions sur les données récentes de la recherche en matière de didactique et de pédagogie (Gouvernement du Québec, 2001, p. 79).

Il doit également adapter les savoirs à enseigner et pour enseigner à la situation didactique à la suite d'une analyse fine des contingences du contexte et des caractéristiques des élèves, puis les articuler à d'autres types de savoirs (psychopédagogiques, par exemple) et de ressources pour traiter avec succès la situation didactique (Jonnaert et Vander Borght, 2009). On voit combien la compétence de planification est « révélatrice des complexités du métier » (De Kesel, Bouhon, Dufays et Plumat, 2013, p. 10). On perçoit aussi combien toutes les décisions qu'ont à prendre les enseignants au moment de planifier les situations d'apprentissage ont avantage à reposer sur des savoirs professionnels légitimes. Or, Gouin, Hamel et Falardeau (2016) ainsi que Messier, Villeneuve-Lapointe, Guay et Lafontaine (2016) ont fait ressortir que les étudiantes et étudiants de divers programmes de formation des maitres peinent à recourir aux savoirs de référence, construits au cours de la formation théorique, pour fonder leurs propos dans les différents travaux universitaires qu'ils doivent réaliser. Il est alors légitime de se demander s'il en est de même en contexte de stage, et dans quelle mesure les stagiaires en enseignement $\mathrm{du}$ français au secondaire mobilisent des savoirs didactiques issus de leur formation universitaire en didactique pour préparer et piloter leur cours en contexte de stage. Une meilleure connaissance de leurs savoirs didactiques et de leur compétence de planification pourra permettre, au besoin, de réfléchir aux moyens d'offrir des formations didactique et pratique mieux articulées.

\section{Le savoir professionnel « didactique »}

Le savoir professionnel peut être entendu comme l'ensemble des savoirs formalisés propres à une profession et à la réalisation des tâches qu'elle implique (Gauthier et al., 1997). Il peut également être entendu du point de vue de la personne qui exerce ou apprend à exercer la profession (Barth, 2002; Vanhulle, 2009) et concerne alors les savoirs qu'elle construit et mobilise. Ces savoirs sont accessibles par le biais du discours et des pratiques du professionnel.

\section{Les savoirs « didactiques»}

La didactique est une discipline de recherche et d'intervention éducative qui met l'accent sur les "savoirs scolaires, leurs spécificités disciplinaires et les incidences que ces spécificités peuvent avoir sur les apprentissages et l'enseignement » (Delcambre, 2007, cité dans Reuter, Cohen-Azria, Daunay, Delcambre et Lahanier-Reuter, 2007, p.163). On peut considérer que les savoirs «didactiques" regroupent les savoirs à enseigner et des savoirs pour enseigner. 
Les savoirs à enseigner, communément appelés «objets » ou « contenus » d'enseignement, sont les savoirs prescrits. Ils proviennent des disciplines contributives (chimie, histoire, littérature) et de pratiques sociales de référence (Martinand, 2001). Ces savoirs « savants » sont devenus des savoirs à enseigner après avoir été choisis par divers décideurs, puis adaptés pour l'institution scolaire, et ce, par un processus de transposition didactique externe (Chevallard, 1985). Ces savoirs scolaires, présentés dans les programmes d'études, vont encore subir des transformations (transposition didactique interne), notamment lorsque l'enseignant s'en saisit et les présente aux élèves, puis lorsqu'ils deviennent des " savoirs appris » par ces derniers. Pour Roger, Maubant et Caselles-Desjardins (2011) ainsi que pour Gauvin et Boivin (2012), les savoirs scolaires ne sont définissables qu'en considérant toute cette chaine de transposition partant du savoir prescrit jusqu'au savoir effectivement appris.

Les savoirs didactiques pour enseigner concernent les modèles d'actions qui ont été élaborés à partir de l'analyse des propriétés des savoirs à enseigner, du modèle d'apprentissage qui les sous-tend et de l'étude de l'activité enseignante empirique (Gagnon et Dolz, 2009). Ils correspondent en clair aux méthodes, aux techniques ou aux dispositifs d'enseignement qui sont développés en tenant compte des spécificités des savoirs scolaires. Cette définition pourrait mener à distinguer les savoirs pour enseigner issus du domaine de la didactique (par exemple, la dictée « zéro faute » en français) de ceux qui proviennent du champ de la pédagogie (par exemple, l'enseignement explicite) en ce que ces derniers ont été modélisés pour soutenir l'apprentissage de n'importe quels savoirs scolaires (Delcambre, 2007, cité dans Reuter et al., 2007), sans égards aux spécificités intrinsèques de ceux-ci. Parce que la pratique enseignante intrique des modèles d'actions didactiques et pédagogiques, il nous est apparu pertinent, dans le cadre de cette étude, de nous intéresser à tous les savoirs pour enseigner puisque, dans un cas comme dans l'autre, ils visent à favoriser l'apprentissage de savoirs scolaires par les élèves.

\section{Les savoirs didactiques du professionnel enseignant}

Le savoir d'une personne est défini ici comme un système cohérent de savoirs en constante recomposition, nécessairement singulier, car subjectif, lié à des processus d'énonciation (Barth, 2002; Buysse, 2011; Gagnon et Dolz, 2009; Vanhulle, 2009). Dans le cas qui nous concerne, nous nous intéressons aux savoirs spécifiquement didactiques (les savoirs à et pour enseigner) qu'ont construits et que mobilisent des stagiaires en enseignement du français. Ces savoirs professionnels se constituent par l'entremise de l'intégration de savoirs d'expérience, nombreux dans le cas des professionnels enseignants, et de savoirs de référence.

Les savoirs d'expérience sont des "savoirs non formalisés à priori qui s'acquièrent au contact de situations et interactions au sein des milieux de pratique et de l'expérience propre [de l'élève, de l'étudiant, du stagiaire et de l'enseignant] »(Vanhulle, 2009, p. 170). Ces savoirs sont ici considérés comme " premiers » et peuvent prendre la forme de manières d'agir, de représentations, de croyances qui n'ont pas été passées au crible de l'analyse réflexive. Ces savoirs peuvent être ou non énonçables par le praticien puisque ce dernier peut ne pas avoir pleinement conscience des connaissances implicites inscrites dans son activité ou son propos (Borde, 2009; Maurel, 2008; Vermersch, 1996). Des savoirs didactiques d'expérience peuvent donc être mobilisés par le stagiaire lors de la préparation d'une activité d'apprentissage : il va alors s'appuyer sur sa compréhension première du savoir à enseigner, choisir de le présenter en empruntant une modalité d'action observée et vécue en tant qu'apprenant, ou provenant de ses représentations, et ce, sans confronter ses choix aux savoirs didactiques issus de sa formation. 
Les savoirs de référence sont pour leur part des «énoncés incorporés dans des pratiques discursives circonscrites et socialement constituées et reconnues » (Hofstetter et Schneuwly, 2009, cité dans Vanhulle, 2009, p .170) par différents acteurs de la profession enseignante. De type déclaratif, ils sont scripturalisés ou énonçables, et servent à décrire, à comprendre et à orienter les pratiques éducatives (Vanhulle, 2009). Certains sont des savoirs dits "théoriques", ce sont ceux qui sont issus de la recherche et des différentes disciplines contributives (Gagnon et Dolz, 2009). D'autres sont dits « de la pratique ». Ce sont les savoirs pratiques formalisés, fortement contextualisés et acceptés comme tels, parce que considérés comme valables et pertinents par la profession (Buysse, 2000). Tous ces savoirs de référence sont considérés comme « externes » au stagiaire, potentiellement "seconds ", et lui sont accessibles sous diverses formes.

Les savoirs de référence de forme académique réputés être scientifiquement fondés sont « proposés aux étudiants comme des références [crédibles] pour comprendre et concevoir leur profession " (Vanhulle, 2009 , p. 170). On les trouve dans les articles scientifiques, les textes théoriques, les ouvrages de référence, sous forme de schémas et de modèles représentés (Buysse, 2000) et, en principe, dans les discours des formateurs universitaires. Les savoirs de forme institutionnelle sont les savoirs prescrits. Ils sont "proposés comme des principes pour agir en fonction des attentes de la société et de l'employeur» (Vanhulle, 2009, p. 170). Nous pensons ici aux savoirs accessibles dans les différents programmes de formation et d'études et dans les orientations ministérielles (par exemple la Politique ministérielle d'évaluation). Quant aux savoirs vulgarisés ${ }^{1}$, il s'agit de savoirs présentés sous forme de pistes concrètes ou par des explications simplifiées que l'on trouve sur des blogues, dans des revues professionnelles, dans le matériel scolaire ou sous forme de conseils ou de suggestions émis par différents formateurs, dont l'enseignant formateur associé ou le superviseur. Les savoirs didactiques de référence devraient être mobilisés par le stagiaire au moment de préparer un cours : il se réfère alors à des sources qu'il juge crédibles pour préparer la présentation des contenus à enseigner et les modalités d'actions qu'il entend mettre en œuvre pour les faire apprendre.

C'est l'intégration consciente (voire la confrontation) des savoirs didactiques d'expérience et de référence, encadrée par une pratique réflexive, qui permettra au stagiaire de se constituer, au fil de la formation initiale et de l'exercice de la profession, son propre savoir professionnel didactique. En effet, lorsque le savoir d'expérience est appréhendé dans le cadre d'une analyse réflexive, c'est-à-dire lorsque l'acteur prend conscience de ses propres manières de dire et de faire, ainsi que de leurs répercussions sur la situation didactique, ses savoirs d'expérience sont mis à l'épreuve et peuvent alors être retenus, modifiés ou rejetés, et devenir (ou pas) des ressources pertinentes et mobilisables. Ces savoirs d'expérience « réfléchis » apparaissent alors de manière structurée (Barth, 2002; Vygotski, 1925/1994) et s'articulent aux modèles et aux pratiques éprouvés.

\section{Retracer les savoirs didactiques}

Pour retracer les savoirs didactiques que mobilisent les stagiaires en enseignement du français au secondaire au moment de mettre en ouvre leur compétence de planification, nous avons choisi de procéder à une analyse exploratoire et qualitative d'un échantillon restreint de préparations de cours réalisées dans le cadre de stages. Ce qui suit présente le protocole méthodologique utilisé. 


\section{Le recueil et le traitement des données}

En février 2017, nous avons sollicité la participation d'étudiants inscrits dans une formation initiale de quatre ans en enseignement du français au secondaire d'une université québécoise. Dans le cadre de cette formation, un stage par année doit être réalisé. Nous avons ciblé les stagiaires de la deuxième et de la quatrième année de formation puisqu'ils avaient réalisé leur stage annuel ${ }^{2}$ à l'automne 2016. Nous avons demandé aux volontaires de nous faire parvenir, par courriel, la préparation d'un cours produit en stage et représentative, du point de vue du stagiaire, de son travail de planification. Nous leur avons demandé d'anonymiser leur fichier pour préserver la confidentialité de ces données invoquées (Van der Maren, 1996). Dix canevas de préparations de stage II et autant de stage IV ont été retenus sur les vingt-trois reçus, les trois canevas rejetés s'étant avérés incomplets. Les fichiers ont été convertis en format PDF et implantés dans le logiciel ATLAS.ti, un logiciel capable de traiter et de codifier des données qualitatives selon une démarche inductive fortement itérative (Paillé et Mucchielli, 2012).

\section{L'outil de recueil de données}

Les stagiaires en enseignement du français au secondaire de cette université doivent utiliser un canevas uniforme pour préparer les cours qu'ils piloteront lors de leur stage. L'outil, flexible ${ }^{3}$, est destiné à soutenir le développement de la compétence à planifier les activités d'enseignement et d'apprentissage (Gouvernement du Québec, 2001) et à en évaluer la progression. La figure suivante présente l'outil qui se déploie généralement sur deux à trois pages selon le cas :

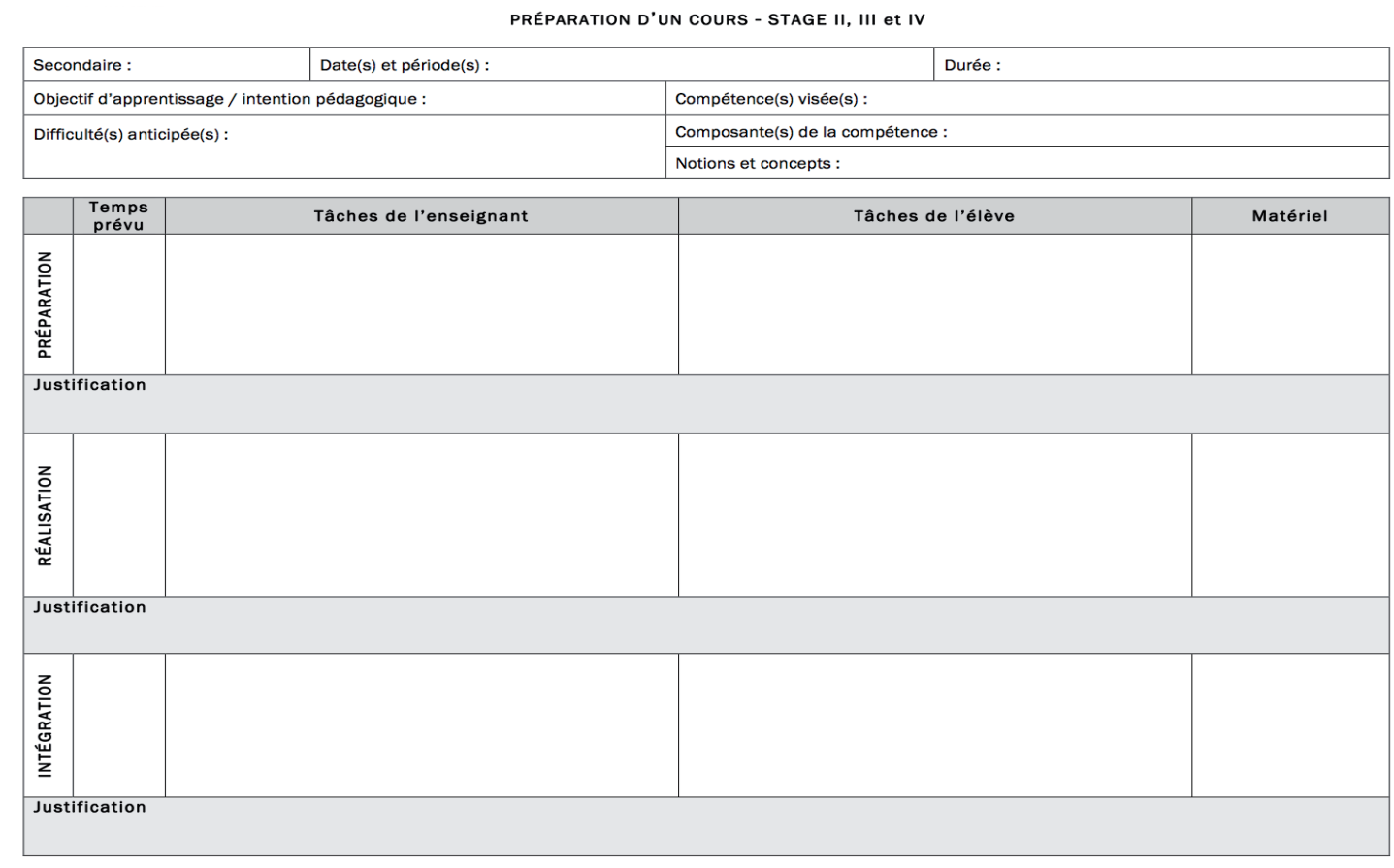

Figure 1

Canevas de préparation d'une leçon. 
Le canevas, conçu par une équipe de superviseurs, comprend les trois temps d'une séance de cours de 75 minutes que sont la préparation, la réalisation et l'intégration des apprentissages (Noverraz, 2016; Tardif, 1992). Les indications fournies en entête ainsi que les descriptions des « Tâches de l'enseignant » et des «Tâches de l'élève » permettent, nous semble-t-il, de retracer, pour chaque stagiaire, ses savoirs didactiques apparents, c'est-à-dire ceux qu'il nomme, définit et décrit dans son canevas de préparation. Toutefois, les raisons qui fondent les choix didactiques des stagiaires restent opaques. Sachant que la conduite de justification donne accès au raisonnement qui sous-tend les choix, aux savoirs qui les fondent, à ce qui est vrai pour un apprenant à un moment donné de l'évolution de ses connaissances (Chartrand, 2013; Forget, 2016), l'équipe de superviseurs a voulu ajouter un espace à la suite de chacune des trois phases afin que le stagiaire puisse justement donner accès aux raisons et aux savoirs qui soustendent les choix des contenus à enseigner et des modalités d'action à mettre en œuvre pour les faire apprendre. Ce sont spécifiquement ces savoirs didactiques « sous-jacents » qui nous intéressent dans le cadre de cet article.

\section{L'analyse des données}

La première étape d'analyse a consisté à repérer les contenus et les modalités d'action qui étaient nommés, définis ou décrits (les savoirs apparents) dans les canevas de préparations de cours, puis à en vérifier la conformité par rapport aux savoirs didactiques de référence de forme académique et institutionnelle (Forget, 2019). La seconde étape d'analyse, sujet du présent article, a consisté à retracer les savoirs sous-jacents, c'est-à-dire ceux qui fondent le choix et l'actualisation des contenus à enseigner et des modalités d'action prévues par les stagiaires. Ces savoirs didactiques sous-jacents ont été repérés dans les justifications fournies. Dans la section qui suit, nous allons détailler, pour chacune des questions de recherche que nous nous sommes posées à propos de ces justifications, les opérations d'analyse réalisées ainsi que les résultats qui en découlent.

\section{Les savoirs didactiques sous-jacents}

Quatre questions ont guidé notre analyse et ont été appréhendées, au cours de l'analyse, l'une à la suite de l'autre :

1. Dans quelle mesure les stagiaires justifient-ils leurs choix « didactiques »?

2. Qu'est-ce qui fait l'objet de justifications « didactiques »?

3. Quels types et quelles formes de savoirs retrouve-t-on dans leurs justifications " didactiques »?

4. Dans quelle mesure leurs savoirs didactiques sont-ils justes?

\section{Dans quelle mesure les stagiaires justifient-ils leurs choix « didactiques »?}

Pour répondre à cette question, nous avons identifié les justifications à proprement parler « didactiques ", c'est-à-dire celles qui placent l'apprentissage de savoirs scolaires au cœur du raisonnement justificatif. Voici trois exemples de justifications qualifiées de « didactiques » (tableau 1) : 


\section{Tableau 1}

Exemples de justifications «didactiques».

\begin{tabular}{l|l}
\multicolumn{2}{c}{ Justifications didactiques } \\
\hline Contenu : type de narrateur & $\begin{array}{l}\text { J'enseigne les types de narrateurs, puisque les élèves devront déterminer le type de } \\
\text { narrateur présent dans un roman [...]. }\end{array}$ \\
\hline Contenu : « intention » de lecture & $\begin{array}{l}\text { J'ai choisi de procéder à un modelage afin que les élèves voient clairement ce à quoi je } \\
\text { m'attends d'eux. Ainsi, ils auront des exemples pour les quatre intentions de lecture } \text { de ce } \\
\text { qu'ils devraient surligner et prendre en note. }\end{array}$ \\
\hline $\begin{array}{l}\text { Contenus : procédés liés au type } \\
\text { de narrateur }\end{array}$ & $\begin{array}{l}\text { L'activité proposée [réécrire un court texte en changeant de type de narrateur] permet aux } \\
\text { élèves de « reconnaitre et utiliser, dans des textes de genre narratif variés, certains procédés } \\
\text { liés au statut de narrateur » (PFÉQ, 2009, p. 154). }\end{array}$ \\
\hline
\end{tabular}

Ces justifications concernent l'apprentissage de savoirs scolaires. En effet, le premier exemple porte sur le choix d'un contenu (types de narrateurs). La raison qui fonde ce choix relève du recours à ce contenu pour la mise en œuvre d'une compétence (lecture) dont le développement est l'une des finalités du cours de français (Gouvernement du Québec, 2009). Dans le second exemple, le stagiaire justifie le choix de recourir au modelage par le fait qu'il permettra aux élèves d'avoir des exemples de ce qu'ils doivent repérer, c'est-à-dire les indices du texte révélant les caractéristiques des personnages, celles du cadre spatiotemporel, les éléments de merveilleux et les figures de style, autant de contenus prescrits dans la Progression des apprentissages (Gouvernement du Québec, 2011). Le troisième exemple porte sur le choix d'une activité (réécriture) justifiée par son potentiel de soutenir l'appropriation de nouvelles connaissances que sont les "procédés liés au statut de narrateur ».

L'ensemble des 20 canevas comportait 88 justifications au total, dont 50 (57\%) peuvent être qualifiées de justifications «didactiques » : 19 (22\%) ont été relevées dans les canevas des stagiaires de deuxième année alors que 31 (35\%) ont été trouvées dans les canevas des stagiaires finissants. Les 38 autres justifications (43\%) n'ont pas fait l'objet d'analyse puisque soit elles ne se sont pas avérées être des justifications «didactiques » au sens où on l'entend dans cet article, soit parce qu'elles ne se sont pas avérées être des justifications. En voici deux exemples (tableau 2):

\section{Tableau 2}

Exemples de justifications rejetées ou de non-justifications.

\begin{tabular}{|c|c|}
\hline \multicolumn{2}{|r|}{ Justifications non retenues } \\
\hline $\begin{array}{l}\text { Justification de l'emploi d'un } \\
\text { outil de gestion de classe }\end{array}$ & $\begin{array}{l}\text { L'utilisation d'un menu comporte certains avantages selon Nault (2016). Tout d'abord il est } \\
\text { un avantage pour l'enseignant, car il constitue un bon aide-mémoire du déroulement de son } \\
\text { cours. Ensuite, il permet aux élèves de situer durant la période. }\end{array}$ \\
\hline $\begin{array}{l}\text { Description de l'activité du } \\
\text { stagiaire }\end{array}$ & $\begin{array}{l}\text { Je modélise les notions apprises et je permets aux élèves de mobiliser les connaissances } \\
\text { apprises. J'active de nouveau le processus réflexif sur les connaissances antérieures. } \\
\text { J'introduis les nouveaux concepts clés de la nouvelle connaissance. Je vérifie l'état de la } \\
\text { compréhension immédiate des élèves. }\end{array}$ \\
\hline
\end{tabular}


Les résultats de cette première étape d'analyse révèlent que les stagiaires de cette étude accordent globalement une importance à peu près équivalente aux choix didactiques et aux questions qui concernent l'encadrement ou la relation maitre-élève. Les stagiaires de quatrième année semblent par ailleurs se soucier davantage des aspects didactiques que leurs condisciples de deuxième année. Cet écart peut s'expliquer par le thème des stages : celui de stage II concerne la communication et la relation pédagogique alors que celui de stage IV porte spécifiquement sur l'acte d'enseigner. Ces résultats montrent également une différence entre les stagiaires de cette étude par rapport à ceux des études citées précédemment (Araujo-Oliveira et al., 2011; Scheepers, 2014), ces derniers étant plus enclins à se préoccuper des aspects relationnels et comportementaux selon ces résultats.

\section{Qu'est-ce qui fait l'objet de justifications « didactiques »?}

La deuxième question nous a menée à identifier les objets des justifications, c'est-à-dire ce sur quoi portent les justifications «didactiques ». Les objets de justification donnent des indices à propos de ce sur quoi les stagiaires portent leur attention, ce qu'ils jugent pertinent de justifier ou ce qui les préoccupe. Pour identifier ces objets, nous avons repris chacune des justifications retenues et avons répondu à la question : qu'est-ce que le stagiaire justifie ici? Le tableau suivant présente ces résultats (tableau 3).

\section{Tableau 3}

Objets des justifications « didactiques».

\begin{tabular}{|c|c|c|}
\hline Le stagiaire justifie... & Stage II & Stage IV \\
\hline Le contenu à enseigner (Notion, stratégie, règle, etc.) & 5 & 5 \\
\hline \multicolumn{3}{|l|}{ Les modalités d'enseignement (ce qu'il entend lui-même faire) dans : } \\
\hline $\begin{array}{l}\text { - La phase de préparation aux apprentissages } \\
\text { (Activer les connaissances antérieures, mise en scène, etc.) }\end{array}$ & 2 & 8 \\
\hline $\begin{array}{l}\text { - La phase de réalisation des apprentissages } \\
\text { (Démarche inductive, enseignement direct, modelage, etc.) }\end{array}$ & 7 & 2 \\
\hline $\begin{array}{l}\text { - La phase d'intégration des apprentissages } \\
\text { (Révision des notions, activité de transfert, etc.) }\end{array}$ & 3 & 3 \\
\hline & 12 & 13 \\
\hline $\begin{array}{l}\text { Les activités dévolues aux élèves } \\
\text { (Exercice, production écrite, discussion, etc.) }\end{array}$ & 1 & 5 \\
\hline $\begin{array}{l}\text { Les interventions évaluatives } \\
\text { (Questionner, vérifier, effectuer un tour de table) }\end{array}$ & 1 & 8 \\
\hline Total & 19 & 31 \\
\hline
\end{tabular}

$\mathrm{Au}$-delà du fait que les stagiaires de quatrième année ont produit deux fois plus de justifications didactiques que leurs collègues de deuxième année, on remarque qu'ils justifient la presque totalité des activités dévolues aux élèves et à l'évaluation, contrairement aux stagiaires de deuxième année apparemment plus préoccupés par leurs propres activités d'enseignement. En effet, c'est la phase de réalisation des apprentissages qui comporte le plus de justifications didactiques dans les canevas de stage II. Ces résultats corroborent ceux de l'analyse des savoirs apparents (Forget, 2019), qui ont mis 
en lumière des modalités d'enseignement répandues chez les stagiaires de deuxième année alors que les stagiaires finissants ont davantage opté pour préparer des activités d'apprentissage dévolues aux élèves. Quant aux questions d'évaluation, on peut penser que les stagiaires de deuxième année n'en ont que très peu fait mention puisque leur formation théorique sur la question a lieu au cours de la troisième année du programme.

\section{Sur quels types et sur quelles formes de savoirs fondent-ils leurs justifications didactiques?}

Les objets justifiés étant identifiés, il nous fallait ensuite établir un portrait du type (savoir d'expérience ou de référence) et de la forme des savoirs (académique, institutionnelle, vulgarisée, etc.) qui sont convoqués pour fonder les choix didactiques. Nous avons donc repris chacune des justifications et avons codé les éléments de la justification à partir des catégories de types et de formes de savoirs présentées à la section 2. Voici deux exemples illustrant ce que nous avons codé comme savoirs de référence, puis comme savoir d'expérience (tableau 4) :

\section{Tableau 4}

Exemples de savoirs de référence et d'expérience.

\begin{tabular}{l|l}
$\begin{array}{l}\text { Type: } \\
\text { Savoir de référence }\end{array}$ & $\begin{array}{l}\text { Lizanne Lafontaine écrit : « L'intention de communication est le point de départ du projet. Il s'áait } \\
\text { du premier élément à clarifier avec les élèves, dès le premier cours. Ils doivent comprendre pourquoi } \\
\text { ils communiqueront oralement afin que la situation ait un sens et que le projet ne soit pas réduit à } \\
\text { l'obtention d'une simple note comme dans un exposé oral réalisé dans un contexte d'oral médium } \\
\text { d'enseignement » (Lafontaine, 2007, p. 14). Ainsi, dans le cadre du projet d'écriture du conte, les } \\
\text { Forme : Académique }\end{array}$ \\
\hline $\begin{array}{l}\text { élèves avaient des destinataires réels, ce qui rend la tâche beaucoup plus concrète pour eux. } \\
\text { Savoir d'expérience }\end{array}$ & $\begin{array}{l}\text { Comme les élèves [du stage] ont beaucoup de difficulté avec l'écriture de l'introduction, je trouvais } \\
\text { pertinent de demander aux élèves de commencer par trouver le sujet amené, le sujet posé et le sujet } \\
\text { divisé qui sont présents [dans des textes fournis]. En ayant plusieurs modèles, les élèves comprennent } \\
\text { davantage ce que l'on attend d'eux. }\end{array}$ \\
\hline Forme: Observation
\end{tabular}


$\mathrm{Au}$ total, 65 différents savoirs ont été relevés dans les 50 justifications didactiques, certaines en contenant plus d'un. Les résultats sont présentés dans le tableau suivant (tableau 5) :

\section{Tableau 5}

Types et formes de savoirs sur lesquels se fondent les justifications.

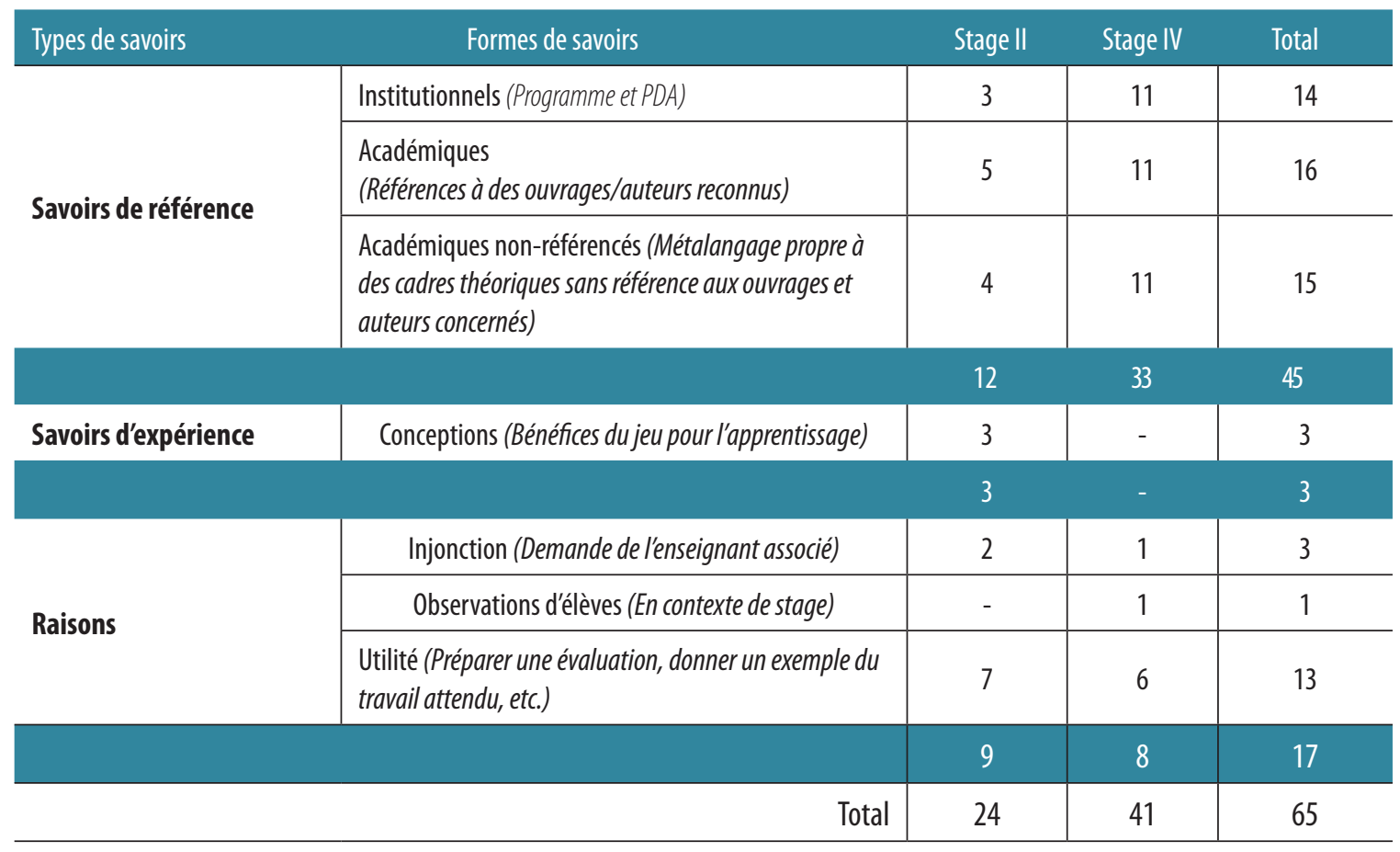

On constate que $70 \%(45 / 65)$ des éléments convoqués à l'appui des justifications sont des savoirs de référence. Cela peut s'expliquer par la consigne qui demandait aux stagiaires de convoquer des savoirs crédibles pour fonder leurs choix et établir des liens avec les cours dits théoriques. Par ailleurs, s'ils s'appuient sur des savoirs académiques (16/65) et institutionnels (14/65) dans une proportion appréciable, la moitié des savoirs académiques ne sont pas accompagnés de la référence à l'auteur ou à l'ouvrage source, alors que le métalangage utilisé dans les justifications évoque un savoir de référence. Par exemple, nous avons considéré que la justification suivante s'appuie sur un savoir de référence puisqu'elle évoque la notion de transfert, mais la source n'est pas indiquée : "Cette activité d'écriture permet un réel transfert des apprentissages puisque les élèves sont amenés à intégrer leurs acquis dans un contexte d'écriture, et non sur une feuille d'exercices». Nous pensons que cette proportion de savoirs de référence, mais non référencés, peut s'expliquer par le fait que les ressources documentaires des étudiants ne sont pas à leur portée en contexte de stage, ce qui rend difficile le référencement. On note aussi que les stagiaires de deuxième année s'appuient moins sur des savoirs de référence, ce qui peut s'expliquer par l'avancement de leur formation. 
Quant aux savoirs d'expérience, on constate qu'ils sont très peu convoqués, et le sont uniquement par les stagiaires de deuxième année. Enfin, l'analyse nous a menée à classer 17 éléments en tant que « raisons ». Ces éléments ne peuvent être considérés comme des savoirs d'expérience dans la mesure où ils constituent des contingences du contexte : injonctions de l'enseignant associé, constats découlant de l'observation des élèves, utilité d'une action pour les étapes à venir, etc. Par exemple, cette justification indique à quoi servira l'activité qui consiste à procéder à une correction en plénière : «Je demanderai à chaque équipe de mentionner un élément, [ainsi] je m’assurerai que la majorité des élèves ont compris la tâche qui leur est demandée et qu'ils pourront partager les informations pertinentes lors de la mise en commun en équipe de base ». Bien que cette catégorie ne soit pas définie comme "savoir » à proprement parler, l'appel à des raisons pour justifier ses choix peut s'avérer tout à fait pertinent s'il découle d'une prise d'information à propos des besoins des élèves ainsi que d'une analyse rigoureuse de la situation didactique, ce qui est le cas pour des stagiaires de cette étude.

\section{Dans quelle mesure les savoirs de référence sont-ils justes?}

Enfin, pour estimer la justesse des savoirs didactiques de référence tels qu'ils sont convoqués par les stagiaires, c'est-à-dire leur exactitude, la confrontation des justifications et des descriptions des tâches de l'enseignant et des élèves est incontournable sur le plan méthodologique (Roger et al., 2011). Cette confrontation permet de vérifier si les tâches décrites sont représentatives des savoirs de référence convoqués et, par conséquent, si ces derniers sont véritablement compris par les stagiaires. Pour ce faire, nous avons repris chacune des justifications comportant un savoir didactique de référence afin d'établir si le savoir en question était exact. Puis nous avons pris connaissance de la description de l'activité à laquelle la justification fait référence, et ce, afin de déterminer dans quelle mesure l'activité reflète le savoir de référence évoqué et, par conséquent, si le stagiaire comprend le savoir de référence auquel il se réfère. Le tableau 6 présente des exemples de savoirs de référence compris, puis non compris selon notre analyse : 


\section{Tableau 6}

Exemples de savoir de référence compris et non compris.

\begin{tabular}{|c|c|}
\hline \multicolumn{2}{|r|}{ Savoirs de référence compris } \\
\hline $\begin{array}{l}\text { Justification: } \\
\text { L'activité proposée [écriture } \\
\text { en changeant de type de } \\
\text { narrateur] permet aux élèves de } \\
\text { «reconnaitre et utiliser, dans des } \\
\text { textes de genre narratif variés, } \\
\text { certains procédés liés au statut de } \\
\text { narrateur » (PFÉQ, 2009, p.154). }\end{array}$ & $\begin{array}{l}\text { Activité décrite: } \\
\text { L'enseignant explique aux élèves la suite des choses : « Maintenant que } \\
\text { vous avez lu et commenté le texte d'un pair, vous devez réécrire ce texte } \\
\text { en employant un narrateur participant ». } \\
\text { L'enseignant questionne les élèves à savoir ce qu’on devrait retrouver dans } \\
\text { leur texte. } \\
\text { Les élèves réécrivent le texte reçu en changeant le type de narrateur } \\
\text { (narrateur omniscient }{ }^{\circledR} \text { narrateur participant). }\end{array}$ \\
\hline & Savoirs de référence non compris \\
\hline $\begin{array}{l}\text { Justification: } \\
\text { La suite du cours est toujours } \\
\text { dans l'esprit de l'enseignement } \\
\text { réciproque (Vienneau, 2011), car } \\
\text { les élèves apprennent à utiliser } \\
\text { des stratégies de lecture en } \\
\text { coopérant. }\end{array}$ & $\begin{array}{l}\text { Activité décrite: } \\
\text { Maintenant que tout le monde a terminé de lire, nous allons commencer } \\
\text { I'annotation. } \\
\text { II y a quatre intentions de lecture : 1-caractéristiques des personnages, } \\
\text { 2-cadre spatiotemporel, 3-univers du merveilleux et 4-figures de style. } \\
\text { Chaque équipe sera responsable d'une intention. Vous allez donc en } \\
\text { devenir experts. } \\
\text { Le but de cette activité est de vous entraider à faire une annotation avec } \\
\text { laquelle vous devez vous familiariser afin d'être en mesure de la continuer } \\
\text { seul et de réutiliser cette technique dans d'autres occasions, soit lors } \\
\text { d'autres lectures. }\end{array}$ \\
\hline
\end{tabular}

Dans le premier exemple, le stagiaire a didactiquement raison d'affirmer que son activité permettra aux élèves de reconnaitre et d'utiliser certains procédés liés au statut du narrateur. Dans le second exemple, le stagiaire se méprend sur le dispositif d'enseignement réciproque. En effet, l'activité décrite ne correspond pas à ce que l'on appelle en didactique de la lecture «l'enseignement réciproque ». Le tableau 7 présente les résultats de cette confrontation entre les justifications et les descriptions d'activités : 


\section{Tableau 7}

Confrontation entre les savoirs de référence convoqués et les activités décrites.

\begin{tabular}{l|l|c|c}
\multicolumn{2}{|r}{} & Justifications & Activités \\
\hline \multirow{4}{*}{ Stage 2} & Exactes & 8 & 2 \\
\cline { 2 - 4 } & En partie exactes & 3 & 3 \\
\cline { 2 - 4 } & Inexactes & 1 & 7 \\
\hline \multirow{3}{*}{ Stage 4} & Exactes & 18 & 16 \\
\cline { 2 - 4 } & En partie exactes & 12 & 6 \\
\cline { 2 - 4 } & Inexactes & 3 & 11 \\
\hline
\end{tabular}

L'analyse fait ressortir une amélioration entre le stage II et le stage IV en ce qui concerne la quantité de savoirs didactiques de référence (12 en stage II contre 33 en stage IV) convoqués dans les justifications et leur compréhension. En effet, si huit justifications comportent des savoirs de référence exactes dans les préparations de cours de stage II, deux activités seulement se sont révélées représentatives de ces savoirs, indiquant ainsi une bonne compréhension de ces derniers. On observe par ailleurs que 10 activités ( 3 en partie exactes et 7 inexactes) sur les 12 analysées ne reflètent pas un savoir didactique de référence qui semble compris par les stagiaires. Ainsi peut-on avancer que les stagiaires de deuxième année possèdent un certain métalangage didactique à propos des modalités d'enseignement et d'apprentissage, mais que ce métalangage ne s'adosse pas encore à une compréhension juste de ces savoirs didactiques, comme le montre l'exemple « non compris » du tableau 6.

Dans les préparations de stage IV, 18 justifications comportent des savoirs de référence exacts, et 16 d'entre eux s'avèrent correctement représentés dans les activités décrites correspondantes, ce qui indique une meilleure compréhension des savoirs didactiques par ces stagiaires. On note tout de même que près de la moitié des justifications (12 en partie exactes et 3 inexactes) et des activités (6 en partie exactes et 11 inexactes) révèlent une mécompréhension du savoir didactique de référence convoqué, ce qui indique que la maitrise des savoirs didactiques, pourtant choisis par les stagiaires eux-mêmes, reste toujours à parfaire au terme de leur formation.

\section{Discussion}

Cet article rend compte d'une étude exploratoire visant à retracer les savoirs didactiques que des stagiaires mobilisent au moment de préparer leur cours. À la lumière des résultats, il est raisonnable de penser que, bien qu'une augmentation notable s'observe dans la mobilisation de savoirs didactiques de référence entre les stages II et IV, un décalage entre ces savoirs didactiques de référence et la compréhension qu'en ont les stagiaires persiste au terme de la formation. Ce décalage montre, à notre avis, une difficulté à s'approprier des savoirs didactiques de référence, qui peut prendre source dans le processus de confrontation des savoirs d'expérience (et des représentations) des stagiaires avec ces savoirs de référence offerts dans le cadre de la formation didactique. Selon nous, cette difficulté peut 
en partie s'expliquer par des formations didactique et pratique mal arrimées, causées par le modèle d'alternance sur lequel sont construits les programmes de formation des maitres. Pour que les stagiaires s'approprient les savoirs didactiques de référence, pour que ces derniers soient "réinterprétés et mis en œuvre dans une logique d'action »(Gagnon, 2007, p. 142), l'arrimage des pratiques de formation professionnelle profiterait d'être accru. En effet, rappelons que le stagiaire doit apprendre à analyser des situations didactiques authentiques afin de déterminer quels savoirs didactiques à et pour enseigner sont susceptibles de répondre le mieux aux besoins des élèves, considérant leurs caractéristiques (Jonnaert et Vander Borght, 2009). Or, la maitrise des savoirs didactiques peut difficilement être assurée par une formation théorique non ancrée dans des contextes authentiques, ce qui nous incite à réfléchir aux moyens de mettre en place des dispositifs de formation mieux à même d'arrimer la formation didactique à ce qu'offrent déjà les stages : des contextes authentiques de développement des compétences professionnelles.

\section{Notes}

1 Précisions que, pour nous, la qualité d'un savoir n’est pas tributaire de la forme (académique, institutionnelle ou vulgarisée) dans laquelle il a été repéré ou présenté : un savoir académique peut être devenu obsolète alors qu'un savoir vulgarisé peut s'avérer très opérationnel et scientifiquement fondé.

2 Dans le cadre de cette formation initiale, le stage II dure 5 semaines consécutives et s'échelonne de la mi-novembre à la mi-décembre tandis que le stage IV dure 8 semaines consécutives et s'échelonne de la mi-octobre à la mi-décembre.

3 Les étudiants utilisent le document en format .docx, ce qui leur permet une flexibilité non seulement sur la densité de la description, mais aussi sur l'enchainement des trois temps de l'apprentissage et leur articulation avec d'autres cours ou d'autres activités comme les routines de classe ou les évaluations.

4 Pour déterminer si un savoir didactique apparait « juste », nous avons utilisé les prescriptions ministérielles ainsi que les cadres en vigueur dans le domaine de la didactique du français en grammaire, en lecture, en écriture et en communication orale. Lorsque le savoir était accompagné d'une référence, celle-ci a pu être consultée, au besoin.

5 Les quatre intentions de lecture correspondent aux caractéristiques des personnages, à celles du cadre spatiotemporel, aux éléments de merveilleux et aux figures de style, autrement dit, au repérage de quatre types de connaissances sur les textes.

\section{Références}

Araujo-Oliveira, A., Lisée, V., Lenoir, Y. et Maubant, P. (2011). Planifications des savoirs d'enseignement chez de futures enseignantes du primaire au Québec : résultat d'une enquête par entrevues. Dans P. Maubant (dir.), Enjeux de la place des savoirs dans les pratiques éducatives en contexte scolaire (p.129-155). Québec, QC : Presses de l'Université du Québec.

Barth, B.-M. (2002). Le savoir en construction. Paris : Retz.

Borde, F. (2009). Psycho-phénoménologie. Expliciter, (79). Repéré à https://www.grex2.com/assets/files/expliciter/expliciter 79 mars 2009 final.pdf

Buysse, A. (2000). Les mécanismes inductifs et déductifs dans la genèse des savoirs des enseignants en formation. Dans P. Maubant, J. Clénet et D. Poisson (dir.), Débats sur la professionnalisation des enseignants. Les apports de la formation des adultes. (p. 267-307). Québec, QC : Presses de l'Université du Québec. 
Buysse, A. (2011). Une modélisation des régulations et de la médiation dans la construction des savoirs professionnels des enseignants. Dans P. Maubant et S. Martineau (dir.), Fondements des pratiques professionnelles des enseignants. (p. 243284). Ottawa, ON : Presses de l'Université d'Ottawa.

Chartrand, S.-G. (2013). Enseigner à justifier ses propos de l'école à l'université. Correspondance, 19(1). Repéré à http://correspo.ccdmd.qc.ca/Corr19-1/2.html

Chevallard, Y. (1985). La transposition didactique. Du savoir savant au savoir enseigné. Grenoble : La Pensée sauvage.

De Kesel, M., Bouhon, M., Dufays, J.-L. et Plumat, J. (2013). La planification des apprentissages. Comment les enseignants préparent-ils leur cours? Louvain-La-Neuve : Presses universitaires de Louvain.

Forget, M.-H. (2016). Loral " pour soi » : tactiques automédiatrices pour l'élaboration de justifications écrites. Recherches en didactiques, (21), 39-56. http://dx.doi.org/10.3917/rdid.021.0039

Forget, M.-H. (2019). Savoirs didactiques de stagiaires en enseignement du français au secondaire : que révèlent leurs préparations de cours?. La Lettre de l'AIRDF, (65), 27-34. http://dx.doi.org/10.3406/airdf.2019.2277

Gagnon, C. (2007). Arrimage des pratiques éducatives d'enseignants et de formateurs en entreprise en contexte d'alternance. Études de cas en formation professionnelle agricole. Recherches qualitatives, 27(1), 141-190. Repéré à http:/www.recherche-qualitative.qc.ca/documents/files/Articles Laureats JMVDM/claudia-gagnon.pdf

Gagnon, O. (2014). Rédaction d'une séquence explicative par de futurs enseignants de français au secondaire. Le français aujourd'bui, (184), 55-68. http://dx.doi.org/10.3917/1fa.184.0055

Gagnon, R. et Dolz, J. (2009). Savoirs dans la formation des enseignants de français langue première : une étude de cas sur l'argumentation orale. Dans R. Hofstetter et B. Schneuwly (dir.), Savoirs en (trans)formation. Au cceur des professions de l'enseignement et de la formation (p. 221-244). Bruxelles : De Boeck. http://dx.doi.org/10.3917/dbu.hofst.2009.01.0221

Gauthier, C., Desbiens, J.-F., Malo, A., Martineau, S. et Simard, D. (1997). Pour une théorie de la pédagogie. Québec, QC : Presses de l'Université Laval.

Gauvin, I. et Boivin, M.-C. (2012). La théorie de la transposition didactique : un outil conceptuel pour décrire les savoirs grammaticaux élaborés en classe par les élèves. Bellaterra Journal of Teaching Eं Learning Language Eं Literature, 5(3), 10-24. http://dx.doi.org/10.5565/rev/jt13.474

Gauvin, I., Boivin, M.-C., Duchesne, J., Lefrançois, P., Ouellet, C., Pinsonneault, R. et Simard, D. (2017). Les savoirs en grammaire et en didactique de la grammaire de futurs enseignants : une porte d'entrée pour réexaminer la formation à l'enseignement. Dans E. Bulea Bronckart et R. Gagnon (dir.), Former à l'enseignement de la grammaire (p. 69-96). Villeneuve-d'Ascq : Presses universitaires du Septentrion.

Gouin, J.-A., Hamel, C. et Falardeau, É. (2016, août). Soutenir la circulation des savoirs inter-travaux des étudiants-stagiaires en formation initiale en enseignement secondaire. Communication au Colloque de l'AIRDF, Montréal, QC.

Gouvernement du Québec. (2001). La formation à l'enseignement : les orientations, les compétences professionnelles. Repéré à http://www.education.gouv.qc.ca/fileadmin/site web/documents/reseau/formation titularisation/formation enseignement orientations EN.pdf

Gouvernement du Québec. (2009). Programme de formation de l'école québécois : Français, langue d'enseignement, secondaire, $2^{e}$ cycle. Repéré à http://www.education.gouv.qc.ca/fileadmin/site web/documents/dpse/formation_jeunes/PFEQ FrancaisLangueEnseignement.pdf

Gouvernement du Québec. (2011). Progression des apprentissages au secondaire : Français, langue d'enseignement. Repéré à http://www.education.gouv.qc.ca/fileadmin/site web/documents/education/jeunes/pfeq/PDA PFEQ francaislangue-enseignement-secondaire 2011.pdf

Jonnaert, P. et Vander Borght, C. (dir.) (2009). Créer des conditions d'apprentissage. Un cadre de référence socioconstructiviste pour une formation didactique des enseignants. Bruxelles : De Boeck.

Lord, M.-A. et Sauvaire, M. (2014). Former les futurs enseignants du Québec à écrire et à enseigner l'écriture. Le français aujourd'bui, (184), 93-101. http://dx.doi.org/10.3917/1fa.184.0093 
Martinand, J.-L. (2001). Pratiques de référence et problématique de la référence curriculaire. Dans A. Terrisse (dir.), Didactique des disciplines. Les références au savoir (p. 17-24). Bruxelles : De Boeck.

Maurel, M. (2008). La psycho-phénoménologie : Théorie de l'explicitation. Expliciter, (77). Repéré à https://www.grex2.com/assets/files/expliciter/expliciter 77 final.pdf

Messier, G., Villeneuve-Lapointe, M., Guay, A. et Lafontaine, L. (2016). Développement des compétences en littératie universitaire : des résultats de recherche à la mise en place d'un cours de baccalauréat. Language and Literacy, 18(2), 79112. http://dx.doi.org/10.20360/g2x60x

Noverraz, J.-C. (2016). Modèle didactique des trois phases d'une séquence d'enseignement. Repéré à https://www.bdrp.ch/system/files/docs/2016-08-11/modele didactique des 3 phases.pdf

Paillé, P. et Mucchielli, A. (2012). L'analyse qualitative en sciences humaines et sociales (3e éd.). Paris : Armand Collin.

Reuter, Y., Cohen-Azria, C., Daunay, B., Delcambre, I. et Lahanier-Reuter, D. (2007). Dictionnaire des concepts fondamentaux des didactiques. Bruxelles : De Boeck.

Roger, L., Maubant, P. et Caselles-Desjardins, B. (2011). Les pratiques éducatives des enseignants. Entre savoirs d'enseignement, savoirs d'expérience et savoirs professionnels. Dans P. Maubant (dir.), Enjeux de la place des savoirs dans les pratiques éducatives en contexte scolaire (p. 213-248). Québec, QC : Presses de l'Université du Québec.

Scheepers, C. (2014). Les préparations de cours : apprendre à écrire, écrire pour faire apprendre. Le français aujourd'bui, (184), 17-28. http://dx.doi.org/10.3917/1fa.184.0017

Tardif, J. (1992). Pour un enseignement stratégique. L'apport de la psychologie cognitive. Montréal, QC : Éditions Logiques.

Van der Maren, J.-M. (1996). Méthodes de recherche pour l'éducation. Montréal, QC : Presses de l'Université de Montréal.

Vanhulle, S. (2009). Savoirs professionnels et construction sociodiscursive de l'agir. Bulletin suisse de linguistique appliquée, (90), 167-188.

Vermersch, P. (1996). L'entretien d'explicitation. Paris : ESF.

Vygotski, L.-S. (1925/1994). Le problème de la conscience dans la psychologie du comportement. Société française, (50), $35-47$.

\section{Pour citer cet article}

Forget, M.-H. (2020). Sur quels savoirs des stagiaires en enseignement du français au secondaire appuient-ils leurs choix didactiques? Formation et profession, 28(2), 35-50. http://dx.doi.org/10.18162/fp.2020.542 